Pacific Journal of Mathematic 


\title{
QUASI-PROJECTIVE AND QUASI-INJECTIVE MODULES
}

\author{
ANNE KOEHLER
}

\begin{abstract}
This paper contains results which are needed to prove a decomposition theorem for quasi-projective modules over left perfect rings.
\end{abstract}

An $R$-module $M$ is called quasi-projective if and only if for every $R$-module $A$, every $R$-epimorphism $q: M \rightarrow A$, and every $R$-homomorphism $f: M \rightarrow A$, there is an $f^{\prime} \in \operatorname{End}_{R}(M)$ such that the diagram

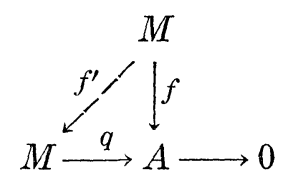

commutes, that is, $q \circ f^{\prime}=f$. An $R$-module $M$ is called quasi-injective if and only if for every $R$-module $A$, every $R$-monomorphism $j: A \rightarrow M$, and $R$-homomorphism $f: A \rightarrow M$, there is an $f^{\prime} \in \operatorname{End}_{R}(M)$ such that the diagram

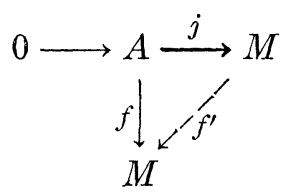

commutes.

The first section of this paper contains results which are needed to prove a decomposition theorem for quasi-projective modules over left perfect rings (Theorem 1.10). This decomposition is a characterization for quasi-projective modules over left perfect rings. A ring is left perfect if a projective cover (the dual concept of injective envelope) exists for every left $R$-module [4, p. 467]. It is known, for example, that left Artinian rings are left perfect [4, p. 467]. Some of the propositions are stated for semiperfect rings which are rings such that every finitely generated module has a projective cover [4, p. 471].

In the second section the decomposition for quasi-projective modules is used to obtain a decomposition for quasi-injective modules over a special class of rings. For these rings this decomposition characterizes quasi-injective modules. This decomposition theorem (Theorem 2.5) is specialized to the cases where the ring is quasi-Frobenius and where it is a finite dimensional algebra.

It will be assumed that all rings have an identity and that the 
modules are unital. Modules will be left R-modules, and homomorphisms will be $R$-homomorphisms unless otherwise stated. When $S$ is the centralizer of ${ }_{R} M$ in the sense of Jacobson [8], the notation will be abused and be written $S=\operatorname{End}_{R}(M)$. Actually, $S$ operates on the right is anti-isomorphic to $\operatorname{End}_{R}(M)$. The radical will mean the Jacobson radical and be denoted by $N$. A direct sum of card $(I)$ copies of $M$ will be written $M^{I}$ unless card $(I)=n<\infty$, and then $M^{n}$ will be used in place of $M^{I}$. Also $\sum_{i=1}^{k} \oplus M_{i}^{g(i)}$ is a direct sum where $M_{\imath}^{g^{(i)}}$ is $g(i)$ copies of $M$, and $g(i)$ can be any cardinal number. If $g(i)=0$, then $M_{\imath}^{g(i)}=0$.

I wish to thank Professor Azumaya who suggested that I investigate quasi-projective modules.

1. Quasi-projective modules. The goal of this section is to prove Theorem 1.10 which is a characterization of quasi-projective modules over left perfect rings. The first proposition to be presented was proved by $\mathrm{Wu}$ and Jans.

Proposition 1.1. Let $R$ be a semi-perfect ring. Then $M$ is a finitely generated, indecomposable, quasi-projective module if and only if $M=R e / J e$ where $e$ is an indecomposable idempotent, and $J$ is an ideal of $R$ [12, Thm. 3.1].

Proposition 1.2. Let $R$ be a semi-perfect ring. If $R e / J e \neq 0$ where $e$ is an indecomposable idempotent and $J$ is an ideal, then $J e=J^{\prime} e$ where $J^{\prime}$ is an ideal contained in the radical $N$.

Proof. The module $N e$ is small in $R e$ [4, p. 473]. Since $R e$ is indecomposable and the projective cover of $R e / N e, R e / N e$ is indecomposable. It is known that $R / N$ is completely reducible if $R$ is semi-perfect [4, Thm. 2.1]. Thus $R \mathrm{e} / \mathrm{Ne}$ is simple, and $N e$ is maximal in $R e$. Now $J e \subseteq N e$ because $N e$ is both maximal and small in $R e$. Let $J^{\prime}=J \cap N$.

Proposition 1.3. If $M$ is quasi-projective and has a projective cover $P \stackrel{\pi}{\longrightarrow} M \longrightarrow 0$ and if $P=\Sigma \oplus P_{a}(a \in I$, and indexing set $)$, then $M=\sum \oplus M_{a}$ and $P_{a} \stackrel{\pi_{a}}{\longrightarrow} M_{a} \longrightarrow 0$ is the projective cover of $M_{a}$ where $\pi_{a}=\pi \mid P_{a}$.

Proof. The proof for the finite case [12, Prop. 2.4] will work here also. 
Proposition 1.4. Let $P_{a} \stackrel{\pi_{a}}{\longrightarrow} M_{a} \longrightarrow 0$ be the projective cover of $M_{a}$ where $a \in I$, an indexing set. If $f\left(\operatorname{Ker} \pi_{a}\right) \subseteq \operatorname{Ker} \pi_{b}$ for every $a, b \in I$ and $f \in \operatorname{Hom}_{R}\left(P_{a}, P_{b}\right)$, then $\Sigma \oplus M_{a}$ is quasi-projective.

Proof. It is sufficient to show that $\Sigma \oplus \operatorname{Ker} \pi_{a}$ is an $\operatorname{End}_{R}\left(\Sigma \oplus P_{a}\right)$ module [12, Prop. 1.1]. Let $q_{c}$ be the projection of $\Sigma \oplus P_{a}$ onto $P_{c}$ and $f \in \operatorname{End}_{R}\left(\Sigma \oplus P_{a}\right)$. We will be done if we show $f\left(\operatorname{Ker} \pi_{b}\right) \subseteq$ $\Sigma \oplus \operatorname{Ker} \pi_{a}$. Let $x \in \operatorname{Ker} \pi_{b}$. Since $q_{a} \circ\left(f \mid P_{b}\right) \in \operatorname{Hom}_{R}\left(P_{b}, P_{a}\right), f(x)=$ $\left(q_{a_{1}} \circ f\right)(x)+\cdots+\left(q_{a_{n}} \circ f\right)(x) \in \operatorname{Ker} \pi_{a}+\cdots+\operatorname{Ker} \pi_{a_{n}} \subseteq \Sigma \oplus \operatorname{Ker} \pi_{a}$.

REMARK. If $I$ is finite or $R$ is left perfect, then the converse is true, that is, if $\Sigma \oplus M_{a}$ is quasi-projective, then $f\left(\operatorname{Ker} \pi_{a}\right) \leqq \operatorname{Ker} \pi_{b}$ for every $a, b \in I$ and $f \in \operatorname{Hom}_{R}\left(P_{a}, P_{b}\right)$.

CoROLlary 1.5. If $M$ is quasi-projective and has a projective cover, then $M^{I}$ is quasi-projective.

Proposition 1.6. If $M_{1}$ and $M_{2}$ are quasi-projective and have projective covers $P_{1}$ and $P_{2}$ which are isomorphic and $M_{1} \oplus M_{2}$ is quasi-projective, then $M_{1} \cong M_{2}$.

Proof. The proof of the dual theorem for quasi-injective modules [7, Prop. 2.4] can be dualized.

Bass has shown [4. p. 473] that if $R$ is a left perfect ring and $P$ is a projective module, then $P=\Sigma \oplus R e_{i}$ where $R e_{i} / N e_{i}$ is simple and $e_{i}$ is an idempotent in $R$. This result will be stated in a different form in the next proposition.

Proposition 1.7. Let $R$ be left perfect. Then $P$ is projective if and only if $P=\sum_{i=1}^{k} \oplus\left(R e_{i}\right)^{g(i)}$ where $R e_{i}$ is the projective cover of a simple module, $e_{i}$ is an indecomposable idempotent, $k$ is the number of non-isomorphic simple modules, and $R e_{i} \nRightarrow R e_{j}$ if $i \neq j$.

Proof. $N e_{i}$ is small in $R e_{i}$ [4. p. 473]. Hence $R e_{i}$ is the projective cover of $R e_{i} / N e_{i}$ and is indecomposable by Proposition 1.3. Since $R / N$ is left Artinian [4, p. 467], and the simple $R$-modules and the simple $R / N$-modules are the same, there are only a finite number of nonisomorphic simple modules. Also, simple modules are isomorphic if and only if their projective covers are isomorphic.

Proposition 1.8. Let $R$ be semi-perfect and $M$ be a finitely generated, quasi-projective module. Then $M$ is indecomposable (nonzero) if and only if $\operatorname{End}_{R}(M)$ is a local ring. 
Proof. (i ) If $M$ is not indecomposable, then $\operatorname{End}_{R}(M)$ has a nonzero idempotent $e$ which is different from the identity. Since neither $e$ nor $1-e$ is a unit, $\operatorname{End}_{R}(M)$ is not a local ring.

(ii) If $M$ is indecomposable, then $M=R e / J e$ where $J^{\prime}$ is an ideal of $R$ and $e$ is an indecomposable idempotent. Thus $M=R^{*} e^{*}$ where $R^{*}=R / J . \quad R^{*}$ is semi-perfect [4, Lemma 2.2]. Since $M$ is indecomposable as an $R^{*}$-module, $e^{*}$ is an indecomposable idempotent. In addition $\operatorname{End}_{R}(M)=\operatorname{End}_{R^{*}}\left(R^{*} e^{*}\right)=e^{*} R^{*} e^{*}$. Finally, $e^{*} R^{*} e^{*}$ is a local ring because $R^{*}$ is semi-perfect and $e^{*}$ is indecomposable [10, p. 76].

LEMMA 1.9. Let $R$ be semi-perfeet and $1=e_{1}+\cdots+e_{n}$ where $e_{1}, \cdots e_{n}$ are orthogona?, indecomposable idempotents. If

$$
R e_{1} / J_{1} e_{1} \oplus R e_{2} / J_{2} e_{2} \oplus \cdots \oplus R e_{m} / J_{m} e_{m}
$$

is quasi-projective where $J_{i}, i=1, \cdots, m$, is an ideal, then there is an ideal $J$ such that $J e_{i}=J_{i} e_{i}$ for $i=1, \cdots, m$.

Proof. The projective cover of $\sum_{1=i}^{m} \oplus R e_{i} / J_{i} e_{i}$ is

$$
\sum_{i=1}^{m} \oplus R e_{i} \stackrel{\pi}{\longrightarrow} \sum_{i=1}^{m} \oplus R e_{i} / J_{i} e_{i} \longrightarrow 0
$$

where $\operatorname{Ker} \pi=\sum_{i=1}^{m} \oplus J_{i} e_{i}$. Since $\operatorname{End}_{R}\left(\sum_{i=1}^{m} \oplus R e_{i}\right)=\sum_{j=1}^{m} \oplus_{i=1}^{m} \oplus e_{i} R e_{j}$, it follows that $J_{i} e_{i} \cdot e_{i} R e_{j} \subseteq J_{j} e_{j}$ for $i, j=1, \cdots, m$ [12. Prop. 2.2]. Let

$$
J=\sum_{i=1}^{m} \oplus J_{i} e_{i} \oplus\left(\sum_{i=1}^{m} \oplus J_{i} e_{i}\right) R\left(1-\sum_{i=1}^{m} e_{i}\right) .
$$

Then $J$ is an ideal because $R=\sum_{j=1}^{n} \oplus \sum_{i=1}^{n} \oplus e_{i} R e_{j}$. Also, $J e_{i}=J_{i} e_{i}$ for $i=1 \cdots, m$.

REMARKS. 1. The proof for Lemma 1.9 remains valid if any subcollection of $e_{1}, \cdots e_{n}$ is used rather than the first $m$ of them.

2. The result that for a semi-perfect ring $1=e_{1}+\cdots+e_{n}$ where $e_{1}, \cdots, e_{n}$ are orthogonal indecomposable idempotents can be found in [10].

Theorem 1.10. Let $R$ be left perfect. Then $M$ is a quasiprojective module if and only if

$$
M=\sum_{i=1}^{k} \oplus\left(R e_{i} / J e_{i}\right)^{g(i)}
$$

where $J$ is an ideal, $e_{1}, \cdots, e_{k}$ are indecomposable idempotents, the number of nonisomorphic simple $R$-modules is $k$, and $R e_{1}, \cdots, R e_{k}$ are the corresponding nonisomorphic projective covers. In addition 
the decomposition is unique up to automorphism.

Proof. (i) Let $M$ be quasi-projective. If $M=0$, then we can choose $J=R$ and be done. If $M \neq 0$, let $P \rightarrow M \rightarrow 0$ be the projective cover of $M$. By Proposition 1.7P $=\sum_{i=1}^{k} \oplus\left(R e_{i}\right)^{g(i)}$ where $R e_{1}, \cdots, R e_{k}$ are the nonisomorphic indecomposable projective covers of all the simple modules. By Proposition $1.3 M=\sum_{i=1}^{b} \oplus \sum_{a \in I_{i}} \oplus M_{a i}$ where card $\left(I_{i}\right)=g(i)$. Proposition 1.6 shows that $M_{a i} \cong M_{b i}$ for every $a, b \in I_{i}$. From Proposition $1.1 M_{a i}=R e_{i} / J_{i} e_{i}$ with $J_{i}$ an ideal and $e_{i}$ an indecompotent. As a result of Lemma 1.9 and the remark following it, there is an ideal $J$ such that $J e_{i}=J_{i} e_{i}$ for $i=1, \cdots, k$.

(ii) Conversely, if $M=\sum_{i=1}^{k} \oplus\left(R e_{i} / J e_{i}\right)^{g(i)}$ with the same notation as in the statement of the theorem and $J \neq R$, then Propositions 1.2 and 1.4 show that $M$ is quasi-projective. If $J=R$, then $M=0$ and is, of course, quasi-projective.

(iii) Uniqueness. Using Proposition 1.8 and a generalized KrullRemark-Schmidt theorem which was proved by Azumaya [1, Thm. 1], we have the following result: if $\sum_{a \in A} \oplus M_{a}$ and $\sum_{b \in B} \oplus M_{b}^{\prime}$ are two decompositions of quasi-projective module into indecomposable, modules, then there is a 1 to 1 , onto mapping $f: A \rightarrow B$ such that $M_{a} \cong M_{f^{\prime}(a)}^{\prime}$.

REMARKs. 1 . Theorem 1.10 is true for semi-perfect rings if $M$ is finitely generated.

2. If $M$ is nonzero in Theorem 1.10 , then $J$ can be chosen is the radical of the ring.

2. Quasi-injective modules. In the first section a decomposition theorem for quasi-projective modules was obtained. The motivation for attempting to prove this proposition came from a paper by Harada on quasi-injective modules [7]. Now Theorem 1.10 will be used to obtain a characterization for quasi-injective modules over left Artinian rings which have a finitely generated, lower distinguished (contains an isomorphic copy of every simple module), and injective module. This class of rings includes quasi-Frobenius rings and finitely generated algebras over commutative Artinian rings [2].

Proposition 2.1. Let $R$ be left Artinian. Then $R$ has a finitely generated, lower distinguished, injective module if and only if the injective envelope of every simple module is finitely generated.

Proof. (Given by G. Azumaya). Assume $Q$ is finitely generated, lower distinguished, and injective. Let $Q_{1}, \cdots, Q_{k}$ be the nonisomorphic injective envelopes of all the simple modules. Then 
$Q=\sum_{i=1}^{k} \oplus Q_{i}^{h(i)}$ where $0 \leqq h(i)<\infty$ [2, Thm. 11, p. 268]. Since $Q$ is lower distinguished, $h(i) \neq 0$ for each $i=1, \cdots, k$. It follows that each $Q_{i}$ is finitely generated. The converse is clear.

Proposition 2.2. If $R$ is left Artinian and has a finitely generated, lower distinguished, injective module, then every indecomposable quasi-injestive module is finitely generated.

Proof. Let $M$ be indecomposable and quasi-injective, and let $Q$ be its injective envelope. $Q$ is indecomposable [7, Proposition 2.3], so it is the injective envelope of a simple module [2, Thm. 1, p. 268]. Hence, $Q$ is finitely generated by Proposition 2.1. Since $R$ is left Noetherian, $M$ is finitely generated.

REMARK. If $R$ is perfect, then every indecomposable quasi-projective module is finitely generated by Proposition 1.7 .

The following proposition was proved by Azumaya for the class of rings in the last two propositions and will be stated without giving his proof.

Proposition 2.3. (Duality Theorem). Let $R$ be a left Artinian ring which has a finitely generated, injective, and lower distinguished module $Q$, and let $S=\operatorname{End}_{R}(Q)$. Then for any finitely generated left $R$-module $X, X^{*}=\operatorname{Hom}_{R}(X, Q)$ is a finitely generated right S-module and $\left(X^{*}\right)^{*}=\operatorname{Hom}_{S}\left(X^{*}, Q\right)={ }_{R} X$. The same is true for finitely generated S-modules [2, Thm. 8, p. 262].

Proposition 2.4. If $R$ is left Noetherian and $M$ is quasi-injective, then $M^{I}$ is quasi-injective.

Proof. Let $Q$ be the injective, envelope of $M$. Since $R$ is left Noetherian, $Q^{I}$ is the injective envelope of $M^{I}$. With this result and a theorem of Johnson and Wong [9, Thm. 1.1], a procedure which is similar to the one found in the proof of Proposition 1.4 can be used to see that $M^{I}$ is quasi-injective.

THEOREM 2.5. Let $R$ be left Artinian and have a finitely generated, lower distinguished, and injective module $Q$. Then $M$ is quasi-injective if and only if

$$
M=\sum_{i=1}^{k} \oplus\left(\operatorname{Hom}\left(e_{i} S / e_{i} J, Q\right)\right)^{g(i)}
$$

where $S=\operatorname{End}_{R}(Q), e_{i}$ is an indecomposable idempotent in $S$ for $i=1, \cdots, k, J$ is an ideal of $S$, the number of nonisomorphic simple 
$R$-modules is $k$, and for $i \neq j e_{i} S \not e_{j} S$. This decomposition is unique up to automorphism.

Proof. If $M=0$, we can choose $J=S$. Thus we will assume that $M$ is a nonzero quasi-injective module. It is known that if $R$ is left Artinian, then it is left Noetherian and has only a finite number of simple $R$-modules. Harada has shown that for left Noetherian rings $M=\sum \oplus M_{a}$ where the $M_{a}$ 's are indecomposable quasi-injective modules and that this decomposition is unique up to automorphism [7, Prop. 2.5]. If $Q_{a}$ is the injective envelope of $M_{a}$, then it is the injective envelope of a simple module (see proof of Prop. 2.2). By the dual theorem of Proposition 1.6 and the result that nonisomorphic simple modules have nonisomorphic injective envelopes, $M=\sum_{i=1}^{k} \oplus M_{i}^{{ }^{g(i)}}$ and $M_{i} \not M_{j}$ for $i \neq j$.

As a result of Proposition 2.2, $M_{i}$ is finitely generated. By the Duality Theorem $\operatorname{Hom}_{R}\left(M_{i}, Q\right)$ is a finitely generated, indecomposable, quasi-projective, right $S$-module. Also, $S$ is right Artinian [2, Thm. 6, p. 259]. Hence, $\operatorname{Hom}_{R}\left(M_{i}, Q\right)=e_{i} S / e_{i} J_{i}$ where $e_{i}$ is an indecomposable idempotent in $S$, and $J_{i}$ is an ideal of $S$. Since $\sum_{g(i) \neq 0} \oplus M_{i}$ is a direct summand of $M$ it is quasi-injetive. It follows that $\operatorname{Hom}\left(\sum_{g(i) \neq 0} \oplus M_{i}, Q\right)=\sum_{g(i) \neq 0} \oplus \operatorname{Hom}\left(M_{i}, Q\right)=\sum_{g(i) \neq 0} \oplus e_{i} S / e_{i} J_{i}$ and is quasi-projective. For $i \neq j \quad M_{i} \not M_{j}$, so $e_{i} S \not e_{j} S$. By Lemma 1.9 and a remark following it we can choose $J_{i}=J$ for $g(i) \neq 0$. In addition $M_{i}=\operatorname{Hom}\left(\operatorname{Hom}\left(M_{i}, Q\right), Q\right)=\operatorname{Hom}_{S}\left(e_{i} S / e_{i} J, Q\right)$.

(ii) Suppose $M=\sum_{i=1}^{k} \oplus\left(\operatorname{Hom}_{S}\left(e_{i} S / e_{i} J, Q\right)^{g(i)}\right.$ with the same notation as in the statement of the theorem. Let $M^{\prime}=\sum_{g(i) \neq 0} \oplus$ $\mathrm{Hom}_{S}\left(e_{i} S / e_{i} J, Q\right)$. Then $\operatorname{Hom}_{R}\left(M^{\prime}, Q\right)=\sum_{g(i) \neq 0} \oplus e_{i} S / e_{i} J$ which is quasi-projective by Theorem 1.10. Thus $M^{\prime}$ is quasi-injective. Let $m=\max \{g(i)\}_{i=1, \ldots, k}$ and $M^{\prime \prime}=\left(M^{\prime}\right)^{m}$. Proposition 2.4 gives us that $M^{\prime \prime}$ is quasi-injective. Therefore the direct summand $M$ is quasiinjective.

Corollary 2.6. Let $R$ be quasi-Frobenius. Then $M$ is quasiinjective if and only if

$$
M=\sum_{i=1}^{k} \oplus\left(\operatorname{Hom}_{R}\left(e_{i} R / e_{i} J, R\right)\right)^{g(i)} .
$$

Proof. $\quad R$ being quasi-Frobenius implies $R$ is left Artinian, self injective, lower distinguished, and finitely generated [2, Thm. 6, p. 259]. Also, $R=\operatorname{End}_{R}(R)$.

COROLLARY 2.7. Let $R$ be a finitely generated algebra over a commutative Artinian ring $K$. Then $M$ is quasi-injective if and only if 


$$
M=\sum_{i=1}^{k} \oplus\left(\operatorname{Hom}_{K}\left(e_{i} R / e_{i} J, F\right)\right)^{g(i)}
$$

where $F$ is the $K$-injective envelope of $K /$ rad $K$.

Proof. $R$ has a finitely generated, lower distinguished, injective module $Q$ such that $R=\operatorname{End}_{R}(Q)$ [2, Prop. 19, p. 273]. The functors $\operatorname{Hom}_{K}(\cdot, F)$ and $\operatorname{Hom}_{R}(\cdot, Q)$ are naturally equivalent for finitely generated $R$-modules [2, Thm. 20, 275].

COROLlaRY 2.8. Let $R$ be a finite dimensional algebra over a field $K$. Then $M$ is quasi-injective if and only if

$$
M=\sum_{i=1}^{k} \oplus\left(\operatorname{Hom}_{K}\left(e_{i} R / e_{i} J, K\right)\right)^{g(i)} .
$$

Proof. $K=F$ in Corollary 2.7 .

\section{REFERENCES}

1. G. Azumaya, Corrections and supplementaries to my paper concerning :KrullRemak-Schmidt's theorem, Nagoya Math. J. 1 (1950), 117-124.

2. —_ A duality theory for injective modules, Amer. J. Math. 81 (1959), 249-278.

3. Completely faithful modules and self-injective rings, Nagoya Math. J. 27 (1966), 697-708.

4. H. Bass, Finitistic dimension and a homological generalization of semi-primary rings, Trans. Amer. Math. Soc. 95 (1960), 466-488.

5. - The Morita theorems, mimeographed notes.

6. S. Eilenberg, Homological dimension and syzygies, Ann. of Math. 64 (1956), 328-336.

7. M. Harada, Note on quasi-injective modules, Osaka J. Math. 2 (1965). 351-356.

8. N. Jacobson, Structure of rings, Amer. Math. Soc., Providence, 1964.

9. R. E. Johnson and E. T. Wong, Quasi-injective modules and irreducible rings, J. London Math Soc., 36 (1961), 260-268.

10. J. Lambek, Lectures on Rings and Modules, Blaisdell Publishing Co., Toronto, 1966.

11. E. Matlis, Injective modules over Noetherian rings, Pacific J. Math., 8 (1958), 511-528.

12. L. E. T. Wu and J. P. Jans, On quasi-projectives, Ill. J. Math. 11 (1967), 439-447.

Received August 19, 1968. This paper is part of the author's thesis at Indiana University and was partially supported by NSF Grant No. GP5799 and an NSF Traineeship.

INDIANA UNIVERSITY

BLOOMINGTON, Indiana 47401

AND

MiAmi University

OXFORD, OHIO 45056 


\title{
PACIFIC JOURNAL OF MATHEMATICS
}

\section{EDITORS}

H. SAMELSON

Stanford University

Stanford, California 94305

C. R. HobвY

University of Washington

Seattle, Washington 98105

\section{J. DugunduI}

Department of Mathematics

University of Southern California

Los Angeles, California 90007

RICHARD ARENS

University of California

Los Angeles, California 90024

\section{ASSOCIATE EDITORS}
E. F. BECKENBACH
B. H. Neumann
F. WOLE
K. Yoshida

\section{SUPPORTING INSTITUTIONS}

\author{
UNIVERSITY OF BRITISH COLUMBIA \\ CALIFORNIA INSTITUTE OF TECHNOLOGY \\ UNIVERSITY OF CALIFORNIA \\ MONTANA STATE UNIVERSITY \\ UNIVERSITY OF NEVADA \\ NEW MEXICO STATE UNIVERSITY \\ OREGON STATE UNIVERSITY \\ UNIVERSITY OF OREGON \\ OSAKA UNIVERSITY \\ UNIVERSITY OF SOUTHERN CALIFORNIA
}

\author{
STANFORD UNIVERSITY \\ UNIVERSITY OF TOKYO \\ UNIVERSITY OF UTAH \\ WASHINGTON STATE UNIVERSITY \\ UNIVERSITY OF WASHINGTON

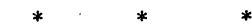 \\ AMERICAN MATHEMATICAL SOCIETY \\ CHEVRON RESEARCH CORPORATION \\ NAVAL WEAPONS CENTER
}

The Supporting Institutions listed above contribute to the cost of publication of this Journal, but they are not owners or publishers and have no responsibility for its content or policies.

Mathematical papers intended for publication in the Pacific Journal of Mathematics should be in typed form or offset-reproduced, (not dittoed), double spaced with large margins. Underline Greek letters in red, German in green, and script in blue. The first paragraph or two must be capable of being used separately as a synopsis of the entire paper. The editorial "we" must not be used in the synopsis, and items of the bibliography should not be cited there unless absolutely necessary, in which case they must be identified by author and Journal, rather than by item number. Manuscripts, in duplicate if possible, may be sent to any one of the four editors. Please classify according to the scheme of Math. Rev. Index to Vol. 39. All other communications to the editors should be addressed to the managing editor, Richard Arens, University of California, Los Angeles, California, 90024.

50 reprints are provided free for each article; additional copies may be obtained at cost in multiples of 50 .

The Pacific Journal of Mathematics is published monthly. Effective with Volume 16 the price per volume (3 numbers) is $\$ 8.00$; single issues, $\$ 3.00$. Special price for current issues to individual faculty members of supporting institutions and to individual members of the American Mathematical Society: $\$ 4.00$ per volume; single issues $\$ 1.50$. Back numbers are available.

Subscriptions, orders for back numbers, and changes of address should be sent to Pacific Journal of Mathematics, 103 Highland Boulevard, Berkeley, California, 94708.

PUBLISHED BY PACIFIC JOURNAL OF MATHEMATICS, A NON-PROFIT CORPORATION

Printed at Kokusai Bunken Insatsusha (International Academic Printing Co., Ltd.), 7-17, Fujimi 2-chome, Chiyoda-ku, Tokyo, Japan. 


\section{Pacific Journal of Mathematics}

\section{Vol. 36, No. $3 \quad$ BadMonth, 1971}

E. M. Alfsen and B. Hirsberg, On dominated extensions in linear subspaces of

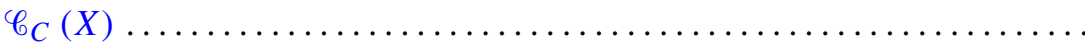

Joby Milo Anthony, Topologies for quotient fields of commutative integral

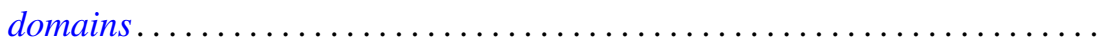

V. Balakrishnan, G. Sankaranarayanan and C. Suyambulingom, Ordered cycle lengths in a random permutation .......................... 603

Victor Allen Belfi, Nontangential homotopy equivalences............... 615

Jane Maxwell Day, Compact semigroups with square roots .............. 623

Norman Henry Eggert, Jr., Quasi regular groups of finite commutative nilpotent algebras .......................................... 631

Paul Erdős and Ernst Gabor Straus, Some number theoretic results ......... 635

George Rudolph Gordh, Jr., Monotone decompositions of irreducible Hausdorff continua .............................................

Darald Joe Hartfiel, The matrix equation $A X B=X \ldots \ldots \ldots \ldots \ldots \ldots . \ldots 69$

James Howard Hedlund, Expansive automorphisms of Banach spaces. II . . . . 671

I. Martin (Irving) Isaacs, The p-parts of character degrees in p-solvable

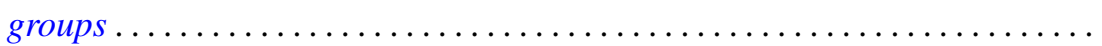

Donald Glen Johnson, Rings of quotients of $\Phi$-algebras ............... 693

Norman Lloyd Johnson, Transition planes constructed from semifield planes....

Anne Bramble Searle Koehler, Quasi-projective and quasi-injective modules.

James J. Kuzmanovich, Completions of Dedekind prime rings as second endomorphism rings...

B. T. Y. Kwee, On generalized translated quasi-Cesàro summability ...

Yves A. Lequain, Differential simplicity and complete integral closure

741

Mordechai Lewin, On nonnegative matrices.

753

Kevin Mor McCrimmon, Speciality of quadratic Jordan algebras ...

Hussain Sayid Nur, Singular perturbations of differential equations in abstract spaces .....................................

D. K. Oates, A non-compact Krein-Milman theorem .

Lavon Barry Page, Operators that commute with a unilateral shift on an invariant subspace...

Helga Schirmer, Properties of fixed point sets on dendrites.

Saharon Shelah, On the number of non-almost isomorphic models of $T$ in a

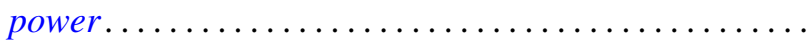

Robert Moffatt Stephenson Jr., Minimal first countable Hausdorff spaces....

Masamichi Takesaki, The quotient algebra of a finite von Neumann algebra 